\title{
miR-574-5p mediates epithelial-mesenchymal transition in small cell lung cancer by targeting vimentin via a competitive endogenous RNA network
}

\author{
YANQIN SUN ${ }^{1,2^{*}}$, YANMEI YI $^{3^{*}}$, SIYUAN GAN $^{1,2^{*}}$, RUIFANG YE $^{1,2^{*}}$, \\ CAILING HUANG ${ }^{1,2}$, MAN LI $^{4}$, JIAN HUANG ${ }^{1,2}$ and YING GUO ${ }^{5}$
}

\begin{abstract}
${ }^{1}$ Department of Pathology, Guangdong Medical University, Dongguan, Guangdong 523808; ${ }^{2}$ Center of
Pathology Diagnosis and Research, Affiliated Hospital of Guangdong Medical University; ${ }^{3}$ Department of Histology and Embryology, Guangdong Medical University, Zhanjiang, Guangdong 524023; Departments of ${ }^{4}$ Pathology and

${ }^{5}$ Organ Transplantation, Zhujiang Hospital, Southern Medical University, Guangzhou, Guangdong 510282, P.R. China
\end{abstract}

Received May 1,2020; Accepted February 12, 2021

DOI: $10.3892 / \mathrm{ol} .2021 .12720$

\begin{abstract}
Numerous studies have suggested that non-coding RNAs mediate tumorigenesis via the epithelial-mesenchymal transition (EMT). However, whether the long non-coding RNA (lncRNA) HOXA transcript at the distal tip (HOTTIP) plays a role in the EMT of small cell lung cancer (SCLC) remains unclear. The results of the present study suggest that HOTTIP-knockdown may lead to a significant increase in E-cadherin expression and a decrease in vimentin (VIM) expression; these proteins are two key markers of EMT. Furthermore, a notable morphological change in SCLC cells with HOTTIP-knockdown was observed: After upregulation of microRNA (miR)-574-5p, the cells exhibited a long, fusiform morphology. Investigating these phenomena further revealed that HOTTIP may participate in EMT by binding to miR-574-5p. In addition, using bioinformatics technology and a dual luciferase reporter assay, it was found that miR-574-5p inhibited VIM expression via direct binding and interaction. In summary, the present results indicate that HOTTIP may be involved in the EMT of SCLC by binding to miR-574-5p, and that miR-574-5p may act through VIM, which is a key marker of EMT.
\end{abstract}

Correspondence to: Dr Ying Guo, Department of Organ Transplantation, Zhujiang Hospital, Southern Medical University, 253 Gongye Avenue, Guangzhou, Guangdong 510282, P.R. China E-mail: guohanjing001@163.com

Dr Jian Huang, Center of Pathology Diagnosis and Research, Affiliated Hospital of Guangdong Medical University, 57 South Renmin Avenue, Zhanjiang, Guangdong 524023, P.R. China E-mail: 18665763598@163.com

${ }^{*}$ Contributed equally

Key words: small cell lung cancer, HOTTIP, miR-574-5p, vimentin, epithelial-mesenchymal transition

\section{Introduction}

Lung cancer remains one of the most fatal diseases in the world, and small cell lung cancer (SCLC) accounts for $>15 \%$ of lung cancer cases (1). The majority of SCLC cases that depend on chemotherapy treatment for surgical resection are less responsive, but almost all cases tend to exhibit subsequent chemoresistance, which results in treatment failure. Therefore, investigating the mechanism of SCLC pathogenesis is crucial.

Non-coding RNAs have no protein-coding potential, and account for $>90 \%$ of transcripts. Among them, microRNAs (miRNAs or miRs) with a length of 19-25 nucleotides (nt) have been extensively studied. Thousands of miRNAs regulate $\sim 30 \%$ of protein-coding genes (2). In addition, long non-coding RNAs (lncRNAs), which are $>200 \mathrm{nt}$ in length, play important regulatory roles in tumorigenesis and tumor progression (3). Previous studies have shown that lncRNAs can regulate the expression of certain oncogenes or cancer suppressor genes by interacting with the lncRNA-miRNA axis, thus affecting the genesis and development of cancer. Furthermore, competitive endogenous RNA (ceRNA) has been confirmed to form an important regulatory network of non-coding RNAs $(4,5)$. Although certain research has been conducted on IncRNA-mediated sponge regulation in several cancer types $(6,7)$, including SCLC, additional studies on this topic are required.

The lncRNA HOXA transcript at the distal tip (HOTTIP), which lies at the $5^{\prime}$ end of the HOXA cluster, has been confirmed to be a key regulator in several cancer types, such as gastric cancer, pancreatic ductal adenocarcinoma, colorectal and ovarian cancer (8). According to previous studies on HOTTIP in tumors, miRNAs usually act as ceRNAs of other genes by targeting HOTTIP. Due to the heterogeneity of different tumors, different miRNAs are usually connected with HOTTIP in different types of cancer $(9,10)$. Our previous studies suggested that HOTTIP participates in the pathogenesis and chemotherapy resistance of SCLC by sponging miR-574-5p and miR-216a-5p $(11,12)$. In the present study, the role of miR-574-5p in the EMT of SCLC and its regulation 
via HOTTIP sponging was explored. In addition, the proliferation- and migration-promoting functions of miR-574-5p in SCLC, and its direct negative regulation of vimentin (VIM) expression, were investigated. Furthermore, the role of the HOTTIP/miR-574-5p/VIM axis in the EMT of SCLC was confirmed, and a therapeutic strategy to regulate the function of oncogenes was suggested.

\section{Materials and methods}

Cell culture. The SCLC cell lines H146, H446, H69 and H69AR cells were obtained from the American Type Culture Collection (ATCC), and were cultured in Gibco RPMI-1640 medium (Thermo Fisher Scientific, Inc.) containing 10 or $20 \%$ fetal bovine serum (Gibco; Thermo Fisher Scientific, Inc.) at $37^{\circ} \mathrm{C}$ and $5 \% \mathrm{CO}_{2}$. Different from the adherent H69AR cell line, the $\mathrm{H} 146$ cell line is a type of semi-suspended and semi-adherent cell line, which makes it more suitable for the observation of cell morphological changes during the EMT process. Since H69AR and H69 cells grow vigorously and exhibit fully adherent growth characteristics according to cell growth characteristics described in ATCC, they are suitable for cell collection, as well as for RNA and protein extraction. According to the needs of the present study, H69, H69AR and H146 SCLC cells were selected for further experiments. Furthermore, the 293T cell line (ATCC), which was were cultured in RPMI-1640 medium containing 10\% fetal bovine serum at $37^{\circ} \mathrm{C}$ and $5 \% \mathrm{CO}_{2}$, was used for the dual luciferase reporter assay.

RNA interference (RNAi) and transfection. HOTTIP small interfering (si)RNA and miR-574-5p inhibitors or mimics (Tables I and II) were obtained from Suzhou GenePharma Co., Ltd. The synthesized HOTTIP and miR-574-5p interference sequences or plasmids as well as their corresponding $\mathrm{NC}$ sequences were transfected into SCLC cells, and the cells with transient down-regulation of HOTTIP and miRNA were obtained. Briefly, $8-10 \times 10^{5}$ cells were inoculated and cultured in a 6-well plate at $37^{\circ} \mathrm{C}$ in a $5 \% \mathrm{CO}_{2}$ incubator. The next day, whe the confluence 70-80\%, Lipofectamine ${ }^{\circledR} 2000$ transfection reagent (Thermo Fisher Scientific, Inc.) was used to transfect $10 \mathrm{nM}$ plasmid or $40 \mathrm{nM}$ siRNA into $1 \times 10^{6}$ cells at $37^{\circ} \mathrm{C}$ for $4-6 \mathrm{~h}$. Subsequently, the medium containing the transfection reagent was discarded and the complete medium was replaced. After 24-48 h, RNA or protein was extracted for subsequent experiments. The lentiviral method was used for stable transfection; the most effective interference sequence of HOTTIP (si-h-HOTTIP-1, confirmed by RT-qPCR) was packaged in an LV3 lentiviral vector (Shanghai GenePharma Co.) for target cell infection experiment, LV3-NC was used as a control, and SCLC cell lines stably silencing HOTTIP were used for further experiments.

Reverse transcription-quantitative PCR (RT-qPCR). RT-qPCR was performed according to the manufacturer's instructions of the TB Green ${ }^{\circledR}$ Premix Ex Taq ${ }^{\mathrm{TM}}$ (Tli RNaseH Plus) (Takara Bio, Inc.), and was used to detect the mRNA expression levels of the related genes in SCLC cells. The extraction of total RNA from cells was performed using TRIzol ${ }^{\circledR}$ reagent (Thermo Fisher Scientific, Inc.). The primers are listed in Table I.
Table I. Primers used for reverse transcription-quantitative PCR analysis.

\begin{tabular}{|c|c|}
\hline Gene & Primer sequence $\left(5^{\prime}-3^{\prime}\right)$ \\
\hline \multirow[t]{2}{*}{ HOTTIP } & Forward: CCTAAAGCCACGCTTCTTTG \\
\hline & Reverse: TGCAGGCTGGAGATCCTACT \\
\hline \multirow[t]{2}{*}{ GAPDH } & Forward: GGGCTGCTTTTAACTCTG \\
\hline & Reverse: TGGCAGGTTTTTCTAGACGG \\
\hline \multirow{2}{*}{ Vimentin } & Forward: AGTCCACTGAGTACCGGAGAC \\
\hline & Reverse: CATTTCACGCATCTGGCGTTC \\
\hline \multirow[t]{2}{*}{ E-cadherin } & Forward: АTTTTTCCCTCGACACCCGAT \\
\hline & Reverse: TCCCAGGCGTAGACCAAGA \\
\hline
\end{tabular}

HOTTIP, HOXA transcript at the distal tip.

SuperScript III Reverse Transcriptase kit (Thermo Fisher Scientific, Inc.) was used to perform the reverse transcription to synthesize cDNA through the following thermal conditions: $30^{\circ} \mathrm{C}$ for $10 \mathrm{~min}, 42^{\circ} \mathrm{C}$ for $30 \mathrm{~min}, 99^{\circ} \mathrm{C}$ for $5 \mathrm{~min}, 5^{\circ} \mathrm{C}$ for $5 \mathrm{~min}$ and $-20^{\circ} \mathrm{C}$ for storage. qPCR was performed under the following conditions: $95^{\circ} \mathrm{C}$ for $30 \mathrm{sec} ; 40$ cycles of $95^{\circ}$ for $5 \mathrm{sec}$ and $60^{\circ}$ for $30 \mathrm{sec}$; and $4^{\circ} \mathrm{C}$ for storage. The mRNA expression was normalized to the expression of GAPDH and calculated using the $2^{-\triangle \Delta C q}$ method. GAPDH was used as a control gene for normalization.

Western blotting and immunofluorescence (IF) staining. Western blotting was performed as previously described (8). Briefly, the samples were collected from SCLC cells using RIPA buffer (Beyotime Institute of Biotechnology). The concentration of proteins was measured using the BCA method (Beyotime Institute of Biotechnology). The proteins (40 $\mu \mathrm{g} /$ lane) were separated by $10 \%$ SDS-PAGE and transferred to PVDF membranes (Thermo Fisher Scientific, Inc.). The membranes were blocked with 5\% non-fat milk powder for $1.5 \mathrm{~h}$ at room temperature. Subsequently, the membranes were incubated with anti-VIM (cat. no. 5741S; dilution, 1:1,000), anti-E-cadherin (cat. no. 8834S; dilution, 1:1,000) and the loading control anti-GAPDH (cat. no. 2118P; dilution, 1:1,000) (all Cell Signaling Technology, Inc.) primary antibodies at $4^{\circ} \mathrm{C}$ overnight. The next day, the membranes were washed with PBS with $0.2 \%$ Tween-20 (PBST) three times and probed with Goat Anti-Rabbit peroxidase-conjugated secondary antibodies (cat. no. A21020; dilution, 1:2,000; Abbkine, Inc.) for $1.5 \mathrm{~h}$ at room temperature. The immunoreactive bands were washed with PBST. Finally, the signal was monitored and visualized using an enhanced chemiluminescence assay (EMD Millipore) and the Odyssey Infrared Imaging system (LI-COR Biosciences). GAPDH was used as an internal control. The intensity of the bands was analyzed by ImageJ software (version 1.8.0; National Institutes of Health).

For IF staining, H146 and H146/miR-574-5p and the corresponding control cells were fixed with $4 \%$ paraformaldehyde at room temperature for $20 \mathrm{~min}$ and blocked with PBS containing 5\% goat serum (cat. no. 16210-064; Gibco; Thermo Fisher Scientific, Inc.). The cells were then washed three times for 5 min with PBS and permeabilized with $0.3 \%$ Triton 
Table II. miR-574-5p mimic/inhibitor/antagomir and HOTTIP siRNA sequences.

\begin{tabular}{ll}
\hline Molecule & \multicolumn{1}{c}{ Sequences (5'-3') } \\
\hline miR-574-5p mimic & UGAGUGUGUGUGUGUGAGUGUGUACACUCACACACACACACUCAUU \\
miR-574-5p inhibitor & ACACACUCACACACACACACUCA \\
Mimics NC & UCUACUCUUUCUAGGAGGUUGUGA \\
Inhibitor NC & UCUACUCUUUCUAGGAGGUUGUGA \\
HOTTIP siRNA & GCUGCUUUAGAGCCACAUAdTdT \\
siRNA NC & UUCUCCGAACGUGUCACGUUU
\end{tabular}

miR, microRNA; siRNA, small interfering RNA; HOTTIP, HOXA transcript at the distal tip; NC, negative control.

$\mathrm{X}-100$ for $1 \mathrm{~h}$ at room temperature. An additional washing step with PBS was performed three times for $5 \mathrm{~min}$, and the cells were incubated with $5 \%$ BSA for $1 \mathrm{~h}$ at room temperature. The samples were incubated overnight at $4^{\circ} \mathrm{C}$ with a primary antibody against VIM (cat. no. 5741S; 1:100; Cell Signaling Technology, Inc.). The samples were subsequently washed with PBS three times for $5 \mathrm{~min}$ and incubated with the secondary antibody (FITC-Labeled Anti-Rabbit IgG; cat. no. CST. 5151p; Cell Signaling Technology, Inc.), and the nuclei were stained with DAPI (Thermo Fisher Scientific, Inc.). An Olympus IX73 microscope was used to capture the fluorescence images. For the quantitative analysis of the intensity of fluorescence expression, the immunofluorescence images were quantitatively analyzed using ImageJ software.

In vitro proliferation assay. According to a standard protocol, plate colony formation experiments were conducted. Single-cell suspension was prepared, and 500 cells were inoculated in a $3.5-\mathrm{cm}$ dish with a diameter of $3.5 \mathrm{~cm}$. The drug concentration was determined according to the $\mathrm{IC}_{50}$ in the treatment group on the next day. Following culture at $5 \% \mathrm{CO}_{2}$, $37^{\circ} \mathrm{C}$ and saturated humidity for 14-21 days, when there were visible clones in the culture dish, the culture was terminated, the supernatant was discarded, and the cells were washed with PBS twice, fixed with pure methanol at room temperature for $10 \mathrm{~min}$ and dyed with $0.4 \%$ crystal violet at room temperature for $10 \mathrm{~min}$. The number of colonies with $>50$ cells was counted under an inverted phase-contrast microscope (CX41, Olympus Corporation). The experiment was repeated three times to obtain mean values (13).

In vitro migration assay. For the wound healing assay, $\mathrm{H} 146$ and H146/si-HOTTIP as well as H146/si-HOTTIP + miR-574-5p cells $\left(5 \times 10^{5}\right.$ per well) were incubated in 6-well plates for $24 \mathrm{~h}$. When the cells were $\sim 100 \%$ confluent, the monolayer was scratched with a $200-\mu 1$ pipette tip. The cells were washed with PBS, and fresh serum-free medium was added. The cells were cultured at $37^{\circ} \mathrm{C}$ with $5 \% \mathrm{CO}_{2}$ and the wound was allowed to heal. Images of the wound were captured at 0 and $24 \mathrm{~h}$. ImageJ software was used to evaluate the wound area, and the wound closure rate was calculated using the following formula: Closure rate $=($ original wound area/final wound area $) /$ original wound area $\mathrm{x} 20$.

A Boyden chamber was used for an in vitro migration assay according to the manufacturer's protocol (BD Biosciences) and previously described techniques (14). Transwell chambers $(8-\mu \mathrm{m}$ pore size; Costar, Inc.) were used to assess the migratory ability of H146 and H146/si-HOTTIP as well as H146/si-HOTTIP + miR-574-5p cells. For the migration assays, $5 \times 10^{4}$ cells were added into the upper chamber. For the invasion assays, Matrigel was dissolved overnight at $4^{\circ} \mathrm{C}$, diluted with serum-free medium at a ratio of $1: 3$, and added at $50 \mu \mathrm{l} /$ well to the top chamber of a Transwell insert. The plate was air-dried in an incubator for $4-5 \mathrm{~h}$ at $37^{\circ} \mathrm{C}$ in a cell incubator. Subsequently, $1 \times 10^{5}$ cells were added into the upper chamber precoated with Matrigel (BD Bioscience). A total of $500 \mu \mathrm{l}$ medium with $15 \%$ FBS was placed into the lower chamber. The plate was maintained at $37^{\circ} \mathrm{C}$ in a $5 \% \mathrm{CO}_{2}$ incubator for $48 \mathrm{~h}$. The cells on the lower surface of the membrane were then fixed with $4 \%$ paraformaldehyde $\left(25^{\circ} \mathrm{C}\right.$ for $\left.10 \mathrm{~min}\right)$ and stained with $0.5 \%$ crystal violet $\left(25^{\circ} \mathrm{C}\right.$ for $\left.30 \mathrm{~min}\right)$. The images were captured in three randomly selected fields under an inverted phase-contrast microscope (Olympus, Japan) at x20 magnification.

Dual luciferase reporter assay. The dual luciferase reporter assay was conducted by Suzhou GenePharma Co., Ltd. Detailed information regarding the methodology is provided on the supplier's website. The putative binding site for miR-574-5p and HOTTIP was predicted using the bioinformatics tool starBase (http://starbase.sysu.edu. cn/index.php). The wild-type (WT) and mutated (MUT) HOTTIP 3'-untranslated region (UTR) sequences were designed and constructed. The mature miR-574-5p and its negative control (NC) were co-transfected with HOTTIP 3'-UTR-WT and HOTTIP 3'-UTR-MUT into cells. The fluorescent enzyme activity of the sample was detected by Dual-Luciferase ${ }^{\circledR}$ Reporter Assay System (Promega Corporation). After $48 \mathrm{~h}$, the cells were harvested and detected for luciferase activity. Luciferase co-transfection was used as a standard control.

Statistical analysis. All experiments were conducted in triplicate. The data are expressed as the mean \pm SD. All statistical analyses were performed using GraphPad Prism 7 software (GraphPad Software, Inc.). Statistical significance was analyzed by unpaired Student's t-test, one-way ANOVA and two-way ANOVA. Bonferroni's correction was applied as a post hoc test after ANOVA. $\mathrm{P}<0.05$ was considered to indicate a statistically significant difference. 
A

\begin{tabular}{|l|l|l|l|}
\hline Gene symbol & H69 cell line & H69AR cell line & Fold-change \\
\hline VIM & 1.162 & 88.304 & 96.4872 \\
\hline TWIST & 0.276 & 0.349 & 2.332 \\
\hline SNAIL & 1.837 & 0.034 & 0.0367 \\
\hline$\beta$-catenin & 0.840 & 0.110 & 0.0806 \\
\hline E-cadherin & 0.718 & 0.146 & 0.2494 \\
\hline
\end{tabular}

B

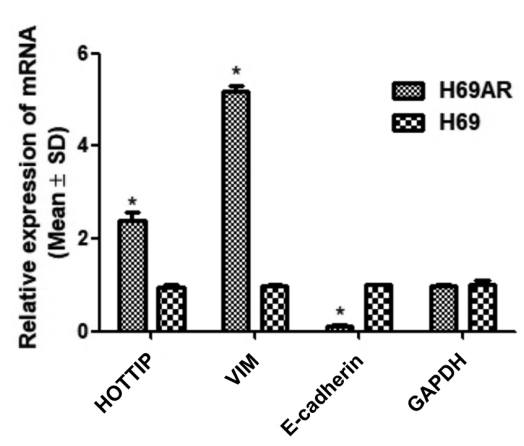

D

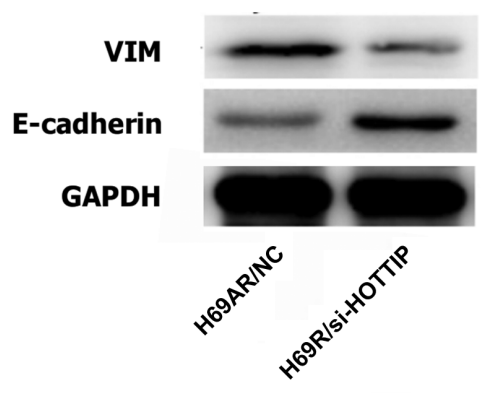

C

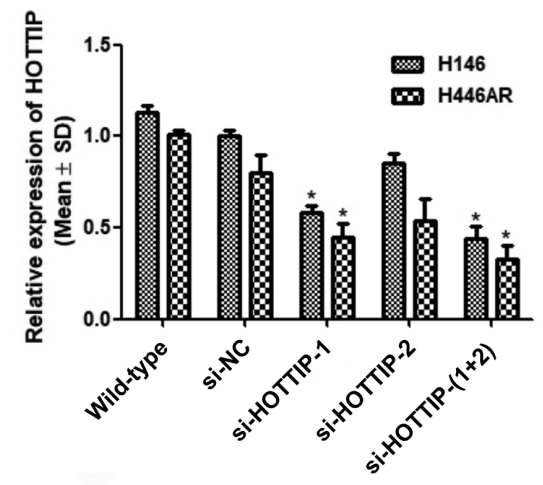

$\mathbf{E}$

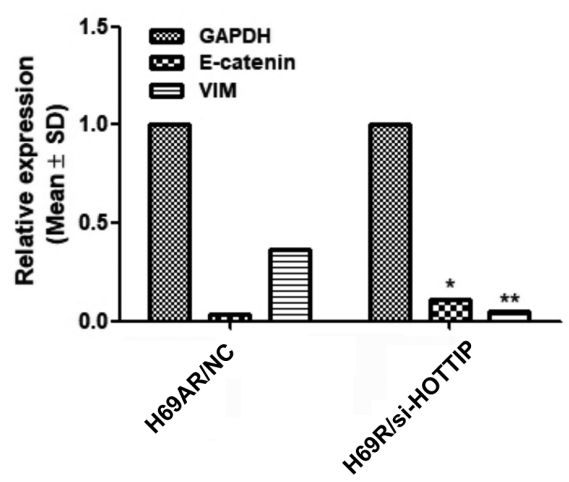

Figure 1. Microarray assay suggests that several EMT markers may be involved in SCLC. (A) Relative expression of five EMT markers, as determined by microarray assay $\left(\times 10^{3}\right)$. (B) The expression of VIM and E-cadherin in H69 and H69AR cell lines was examined by RT-qPCR ("P<0.05 vs. H69). (C) Transfection effect of HOTTIP RNAi sequences in SCLC cell lines ("P $<0.05$ vs. NC or Wild-type) (D) Western blot assay showed the effects of HOTTIP-knockdown on the expression of VIM and E-cadherin proteins. (E) Histogram of the western blot bands shown in Fig. 1F ("P<0.05 and" P $<0.01$ vs. GAPDH). EMT, epithelial-mesenchymal transition; SCLC, small cell lung cancer; RT-qPCR, reverse transcription-quantitative PCR; VIM, vimentin; RNAi, RNA interference; HOTTIP, HOXA transcript at the distal tip; si, small interfering; NC, negative control.

\section{Results}

IncRNA HOTTIP may be involved in the EMT of SCLC. The present study suggested that several EMT markers showed differential expression between the H69 and H69AR cell lines (Fig. 1A). The present study investigated the expression of two key genes in EMT, VIM and E-cadherin, as well as that of HOTTIP, in the H69 and H69AR cell lines. It was found that VIM was highly expressed in H69AR cells, whereas E-cadherin was significantly decreased in H69AR cells (Fig. 1B), and part of HOTTIP-related data has been published (12). Notably, the transfection effect of HOTTIP RNAi sequences as well as that of lentivirus-packaged HOTTIP RNAi sequences were verified by immunofluorescence assay and RT-qPCR; following the establishment of four cell lines with stable and low expression of the HOTTIP gene, these cell lines were used for subsequent in vitro and in vivo experiments (Fig. 1C) (12). To analyze whether there was a difference between the expression of HOTTIP and EMT markers, VIM and E-cadherin, western blotting was used for protein detection (Fig. 1D and E). The results suggested that HOTTIP knockdown could decrease VIM expression 
A

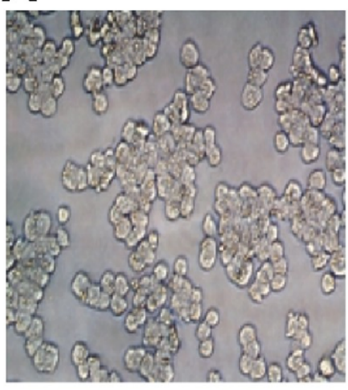

E

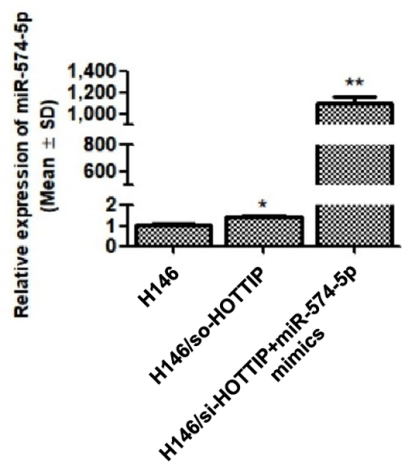

G

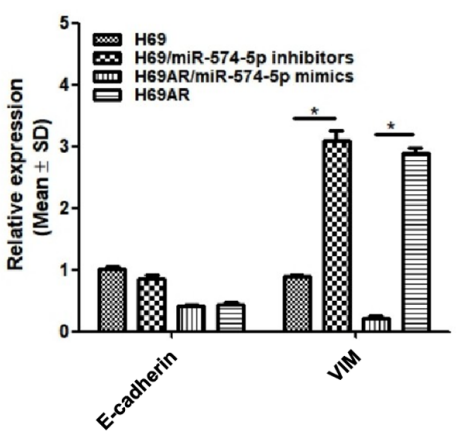

C

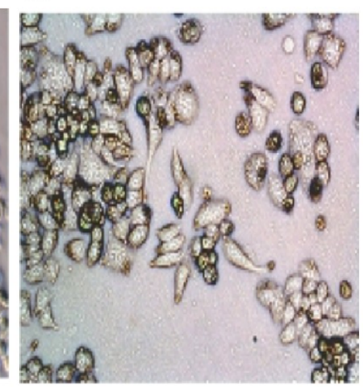

F

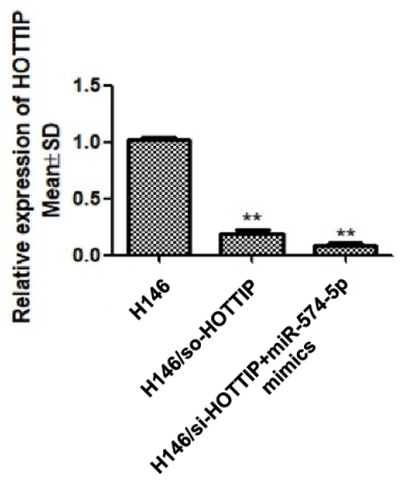

H

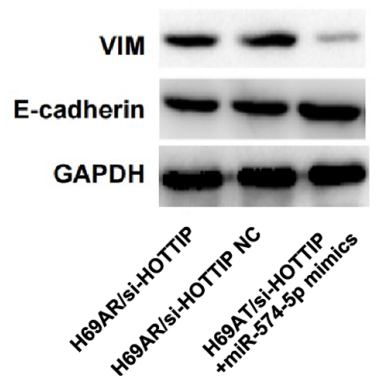

D
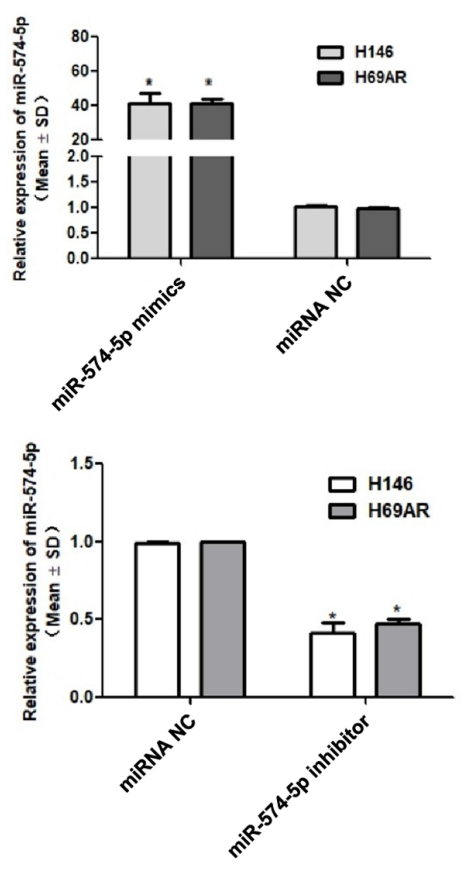

I

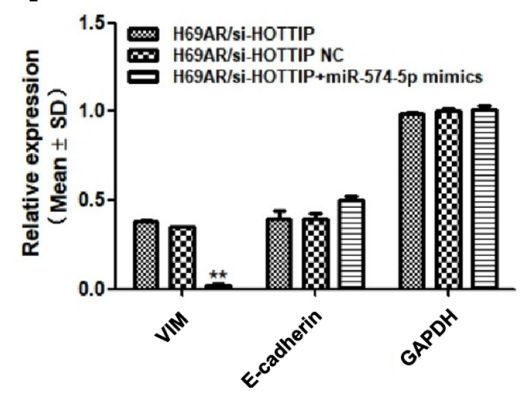

Figure 2. miR-574-5p may induce epithelial-mesenchymal transition via HOTTIP sponging. (A) Morphology of H146 cells (magnification, x200). (B) Morphology of H146/si-HOTTIP cells (magnification, x200). (C) Morphology of H146/si-HOTTIP cells after transfection with miR-574-5p mimics (magnification, x200). (D) Transfection efficiency of miR-574-5p mimics and inhibitor in H146 and H69AR cells. (E) Expression of miR-574-5p in H146 cells transfected with miR-574-5p mimics ( ${ }^{* *} \mathrm{P}<0.01$, ${ }^{*} \mathrm{P}<0.05$ vs. H146). (F) Expression of HOTTIP in H146 cells transfected with miR-574-5p mimics $\left({ }^{* * *} \mathrm{P}<0.01\right.$ vs. H146). (G) Regulatory effects of miR-574-5p on VIM and E-cadherin ("P<0.05). (H) The effects of HOTTIP silencing and miR-574-5p upregulation on VIM and E-cadherin expression were evaluated by western blotting. (I) Histogram of the western blotting results of Fig. $2 \mathrm{H}\left({ }^{* *} \mathrm{P}<0.01\right.$ vs. H69AR or H69AR/si-HOTTIP). HOTTIP, HOXA transcript at the distal tip; VIM, vimentin; miR, microRNA; si, small interfering; NC, negative control.

but increase E-cadherin expression; thus, HOTTIP may be involved in the EMT of SCLC.

HOTTIP may participate in EMT by binding to miR-574-5p . As described in a previous study (12), H146/si-HOTTIP cells were established by lentiviral vector transfection (Fig. 2B). By subsequent transfection with miR-574-5p mimics, H146/si-HOTTIP + miR-574-5p cells were established (Fig. 2C), and their cell morphology was observed under a microscope. In contrast to H146 cells (Fig. 2A), which had an ovular and semi-adherent morphology, the cells in the H146/si-HOTTIP group were smaller and agglomerated into spheres (Fig. 2B), whereas the cells in the H146/si-HOTTIP + miR-574-5p group exhibited a spindle, fusiform and fibroblast-like morphology
(Fig. 2C). The transfection efficiency of miR-574-5p mimics and inhibitor in H146 and H69AR cells was verified (Fig. 2D). The present study next intended to explore the mechanism of the aforementioned morphological changes and to confirm successful transfection. RT-qPCR was used to verify the effects of transfection, and it was found that HOTTIP-knockdown did not affect miR-574-5p expression (Fig. 2E), whereas transfection with miR-574-5p mimics further reduced HOTTIP expression in H146/si-HOTTIP cells (Fig. 2F).

Based on the aforementioned results, in order to determine whether HOTTIP induced EMT by binding to miR-574-5p, the expression of VIM and E-cadherin in H69AR and H69 cells was determined following transfection with miR-574-5p mimics. It was found that, compared with that of the corresponding 
A

hso-miR-574-5p/VIM Alignment

\begin{tabular}{|c|c|}
\hline $\begin{array}{l}\text { 3' ugugugagugugUGUGUGUGAGu 5' hea-miR-574-5p } \\
:: \text { IIIIIIIIII } \\
1: 5^{\prime} \text { - }\end{array}$ & $\begin{array}{ll}\text { mirsVR score: } & -1.0631 \\
\text { PhastCons score: } 0.7019\end{array}$ \\
\hline
\end{tabular}

\begin{tabular}{|c|c|}
\hline $\begin{array}{l}\text { 3' ugugugAGUGUGUGUGUGUGAGU 5' has-miR-574-5p } \\
\text { : | III II IIIIII } \\
\text { 138:5' aguueUUAACA-ACCGACACUCe 3' vIM }\end{array}$ & $\begin{array}{l}\text { mirSVR score: } \quad-0.2265 \\
\text { PhastCons score: } 0.7108\end{array}$ \\
\hline
\end{tabular}

B
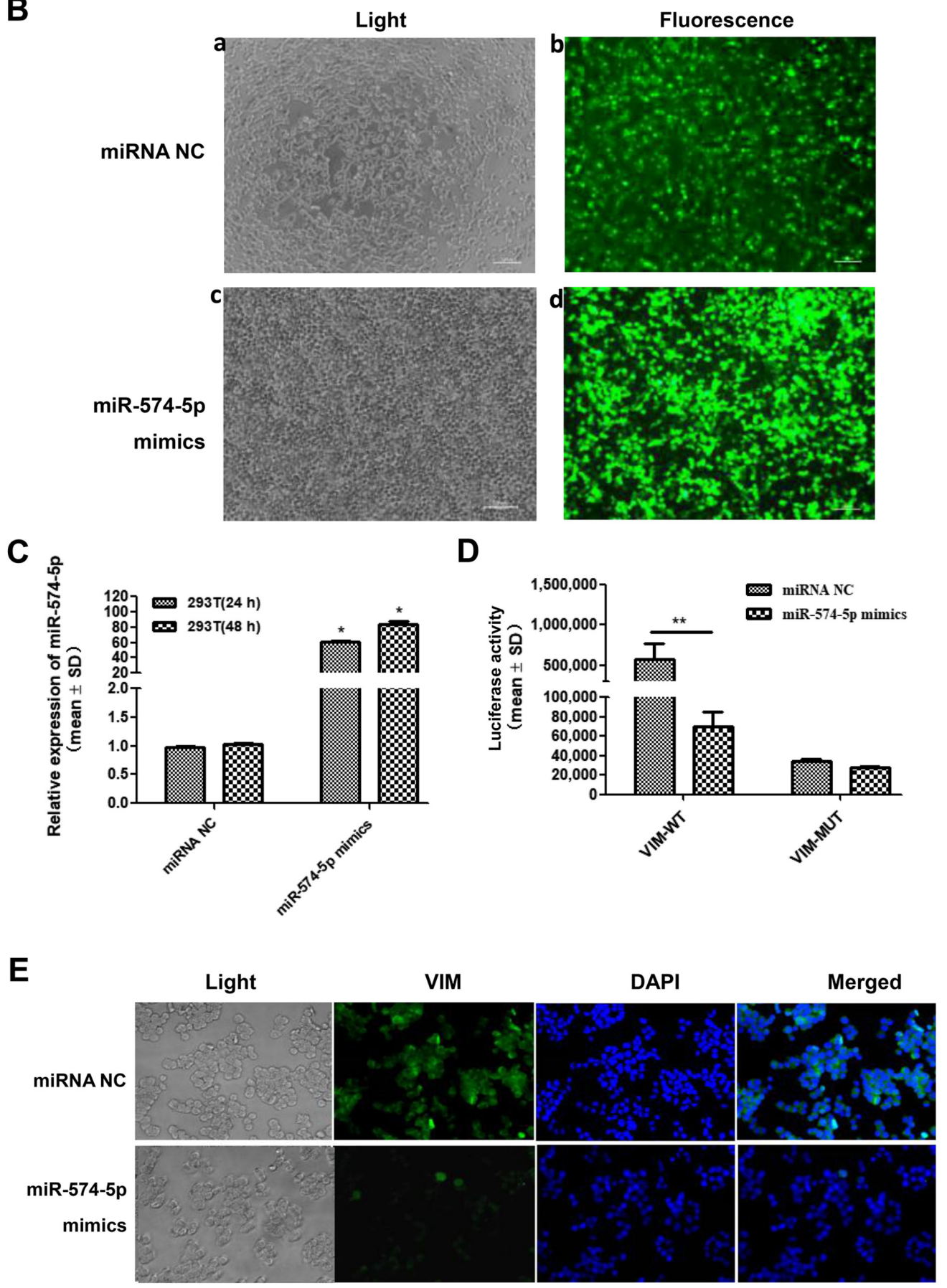

Figure 3. miR-574-5p may regulate the expression of VIM by direct binding. (A) miR-574-5p has two targeted binding areas with VIM according to bioinformatics prediction. (B) Immunofluorescence assay showed that miR-574-5p mimics and NC were successfully transfected into 293T cells (magnification, x20); the transfected cells emitted green fluorescence. (B-a) 293T cell transfection with miR-574-5p NC for $48 \mathrm{~h}$ was observed under a light microscope. (B-b) 293T cells with miR-574-5p NC transfection for $48 \mathrm{~h}$ were observed under a fluorescence microscope. (B-c) 293T cells with miR-574-5p mimics transfection were observed under a light microscope at $48 \mathrm{~h}$. (B-d) 293T cells with miR-574-5p mimics transfection were observed under a fluorescence microscope at $48 \mathrm{~h}$. (C) Reverse transcription-quantitative PCR demonstrated that the miR-574-5p level was increased in 293T cells following transfection with miR-574-5p mimics ( ${ }^{*} \mathrm{P}<0.05$ vs. miRNA NC). (D) Dual luciferase reporter assay showed that miR-574-5p could regulate VIM expression by direct binding $\left({ }^{* *} \mathrm{P}<0.01 \mathrm{vs}\right.$. miRNA NC). (E) miR-574-5p could reduce VIM expression at the protein level, according to the results of immunofluorescence analysis (magnification, $\mathrm{x} 200$ ). VIM, vimentin; miR, microRNA; WT, wild-type; MUT, mutant; NC, negative control. 
A

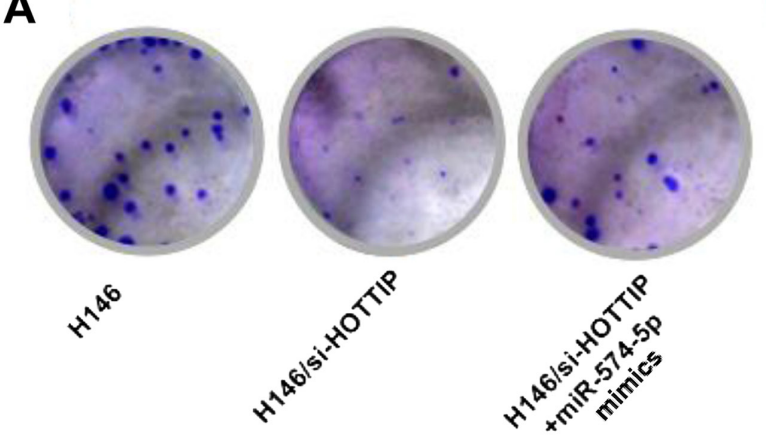

C

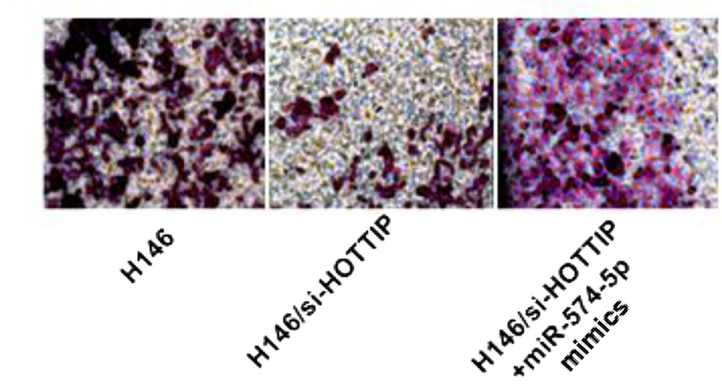

E

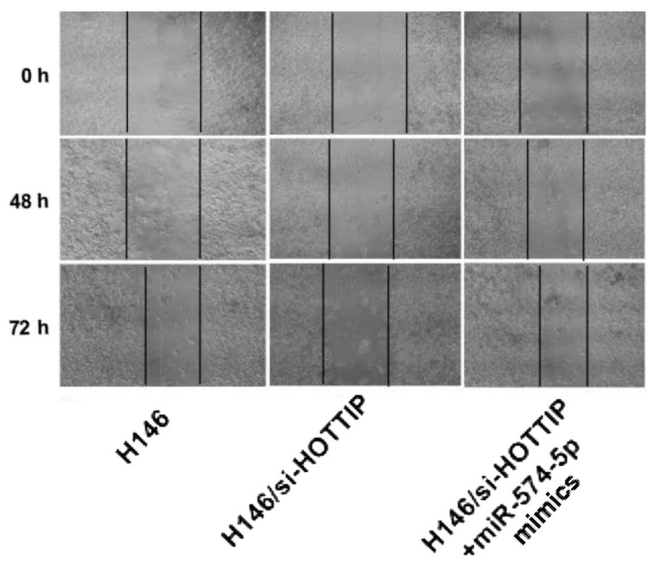

B
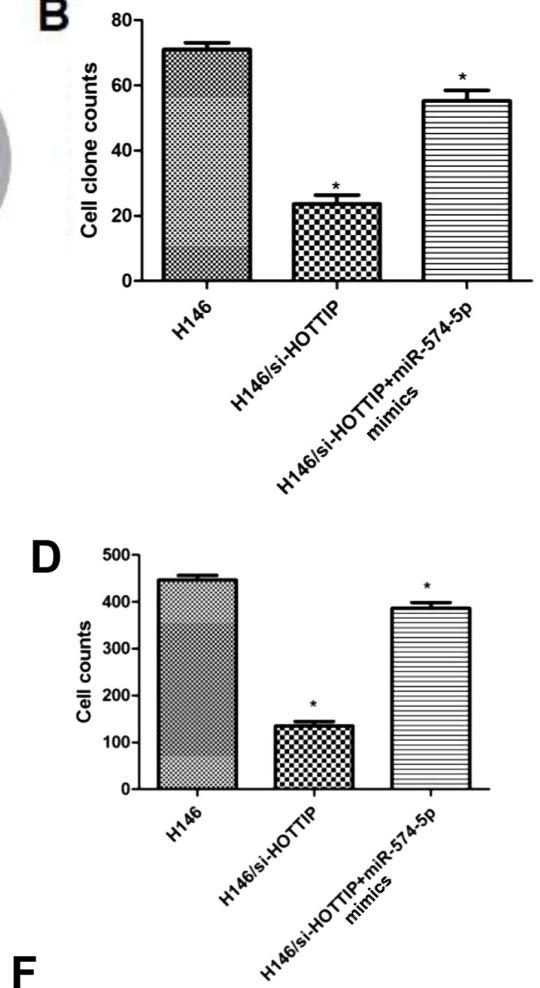

$\mathbf{F}$

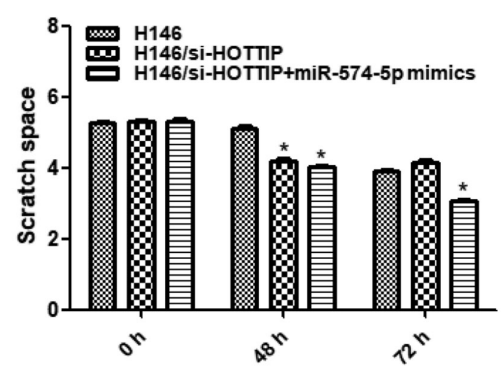

Figure 4. HOTTIP affects colony formation and cell migration abilities by regulating miR-574-5p. (A) Representative images of the colony formation assay (magnification, x20). (B) Differences in colony formation ability between H146, H146/si-HOTTIP and H146/si-HOTTIP + miR-574-5p mimics cells after transfection with miR-574-5p mimics ("P<0.05 vs. H146). (C) Representative Transwell assay images (magnification, $\mathrm{x} 20$ ). (D) Differences of cell migration ability between H146, H146/si-HOTTIP and H146/si-HOTTIP+miR-574-5p mimics cells, as determined by transwell assay ("P<0.05 vs. H146). (E) Representative wound healing assay images (magnification, x20). (F) Differences in cell migration ability between H146, H146/si-HOTTIP and H146/si-HOTTIP+miR-574-5p mimics cells, as determined by wound healing assay ("P<0.05 vs. H146 at 48 or 72 h). miR, microRNA; HOTTIP, HOXA transcript at the distal tip; si, small interfering.

H69AR group, VIM expression in the H69AR/miR-574-5p mimics group was significantly decreased, whereas it was significantly increased in the H69AR/miR-574-5p inhibitor group (Fig. 2G). As the H69AR/miRNA-NC cells were contaminated and RNA could not be extracted, the wild-type group were used as negative control for this experiment. However, changes in E-cadherin expression following transfection were not obvious (Fig. 2G). Therefore, the induction of EMT by HOTTIP sponging of miR-574-5p may be achieved mainly by the regulation of VIM at the mRNA level. Correspondingly, analysis at the protein level by western blotting revealed that the expression of E-cadherin changed only slightly, while VIM expression was significantly decreased in the H69AR/si-HOTTIP + miR-574-5p mimic group compared with that of the H69AR/si-HOTTIP group (Fig. 2H and I). Thus, miR-574-5p regulated the expression of HOTTIP, which has been confirmed in a previous study (12).

VIM is predicted and confirmed to be a target gene of $m i R-574-5 p$. By bioinformatics prediction, two pairable base regions were found in the sequences of miR-574-5p and VIM (Fig. 3A). To evaluate whether their binding was effective, a dual luciferase reporter assay was performed. Fig. 3B shows fluorescence images of plasmid and oligo transfections, which indicated that miR-574-5p mimics and NC were successfully transfected into 293T cells (Fig. 3B), and RT-qPCR demonstrated that the miR-574-5p level was increased in 293T cells following transfection with miR-574-5p mimics (Fig. 3C). It was found that, in 
293T cells, miR-574-5p could target and regulate the expression of VIM (Fig. 3D). Subsequent IF staining confirmed the regulation of VIM protein by miR-574-5p. VIM expression (green fluorescence) in the cytoplasm and nucleus of H146/NC cells differed after transfection with miR-574-5p mimics (Fig. 3E).

HOTTIP may affect colony formation and cell migration by binding to miR-574-5p. To further investigate the effects of HOTTIP binding to miR-574-5p on cell proliferation and invasion, plate colony-forming experiments were carried out. It was found that the colony formation ability of cells was significantly reduced in the H146/si-HOTTIP group compared with that of the H146 group. However, after transfection of H146/si-HOTTIP cells with miR-574-5p mimics, the colony formation ability was restored (Fig. 4A). Fig. 4B shows the quantitative results of the cell colony formation assay.

In a Transwell migration assay, the migration ability of cells in the H146/si-HOTTIP group was significantly reduced compared with that of cells in the non-transfected group, but it was restored by subsequent transfection with miR-574-5p mimics in the H146/si-HOTTIP + miR-574-5p group (Fig. 4C). Fig. 4D shows the quantitative results of cell migration.

Next, a wound healing experiment was conducted to verify the aforementioned results. At $48 \mathrm{~h}$, the wound healing ability of the two treatment groups was poorer compared with that of the control group. However, at $72 \mathrm{~h}$, the H146/si-HOTTIP + miR-574-5p group exhibited the highest wound closure rate (Fig. 4E). Fig. 4F demonstrates the quantitative results of the wound healing assays.

\section{Discssion}

Since the prognosis of SCLC is markedly poor, the study of the pathogenesis and development of SCLC may lead to the identification of new biomarkers and thus aid early detection and treatment. The ceRNA network is a key regulatory mechanism in the pathogenesis and development of numerous tumors, and is a regulatory network involving lncRNAs, miRNAs and their target genes. Our previous study indicated that HOTTIP participates in the pathogenesis, development and chemoresistance of SCLC by sponging miR-574-5p (12). The current study explored whether HOTTIP may play a key role in the EMT of SCLC by sponging miR-574-5p and enhancing VIM expression.

Multiple studies have suggested that HOTTIP acts as an oncogene in esophageal squamous cell carcinoma by inducing EMT via the HOTTIP-miR-30b-HOXA13 axis, in which HOXA13 is a downstream factor of HOTTIP that has been demonstrated to be directly regulated by HOTTIP $(15,16)$. HOTTIP has also been reported to promote EMT in breast cancer and tongue squamous carcinoma through the ceRNA network or other mechanisms $(17,18)$. Previous studies have suggested that HOTTIP and c-Myc may form a positive feedback regulatory loop in osteosarcoma, whereas salinomycin may reduce the development of EMT-mediated multidrug resistance by changing the expression of HOTTIP in gastric cancer cells $(19,20)$. In addition, HOTTIP has been reported to modulate the properties of cancer stem cells via EMT in pancreatic cancer and hepatocellular carcinoma $(21,22)$.

Previous studies on human gastric carcinoma cells have shown that miR-574-3p regulates EMT, as well as cisplatin resistance, by targeting zinc finger E-box-binding homeobox 1 (23); however, the association between miR-574-5p and EMT in cancer remains unclear. The present findings enrich the knowledge on the induction of EMT mediated by non-coding RNA to facilitate tumor metastasis.

Our previous study evaluated HOTTIP, miR-574-5p and the prognosis of patients with SCLC (12). The present study further revealed that HOTTIP may induce the EMT of SCLC by sponging miR-574-5p, and demonstrated that miR-574-5p regulates VIM directly. However, HOTTIP-knockdown also reduced the expression of E-cadherin, another key marker of EMT, although there may not be an obvious regulatory association between miR-574-5p and E-cadherin. HOTTIP may affects the expression of E-cadherin through other mechanisms, such as the Wnt- $\beta$-catenin signaling pathway $(17,24)$ or modifications by salinomycin (19) as reported in other types of cancer.

There are certain limitations in the present study, including the absence of in vivo experiments and the use of only one cell line for certain experiments, and no non-cancerous cell lines used as a negative control. In-depth studies at the tissue level were not conducted, since a suitable model has not been established to analyze the EMT phenomenon in tumor tissues. Future studies should be conducted to confirm the results of the present study in additional cell lines, to verify the association between key molecules and tumor metastasis in vivo, and to further explore the signaling pathways involved in HOTTIP and miR-574-5p.

In conclusion, the present study showed that HOTTIP may participate in EMT by sponging miR-574-5p. In addition, by using bioinformatics technology and a dual luciferase reporter assay, it was confirmed that miR-574-5p probably inhibits the expression of VIM, a key molecule of EMT, through direct target binding. Future studies may focus on the regulation of SCLC metastasis by HOTTIP.

\section{Acknowledgements}

The authors would like to thank Dr Linlang Guo (Department of Pathology, Zhujiang Hospital of Southern Medical University) for the designing guidance in the present study. The dual luciferase reporter assay was performed by Suzhou GenePharma Co., Ltd.

\section{Funding}

The present study was partly supported by the National Natural Science Foundation of China (grant no. 81702285), Funds for the Construction of Basic Medical Disciplines in Guangdong Medical University (grant no. 4SG19047G), Innovation experiment project of Guangdong Medical University in 2020 (grant no.ZZDS007) and Key projects of Guangdong Medical University (grant no. GDMUZ201808).

\section{Availability of data and materials}

The datasets used and/or analyzed in the current study are available from the corresponding author upon reasonable request. 


\section{Authors' contributions}

YS, YY and SG conceived and designed the experiments. $\mathrm{JH}$ and $\mathrm{YG}$ conceived the study. RY and $\mathrm{CH}$ performed the in vitro assays. ML performed the immunofluorescence staining. JH and YG confirm the authenticity of all the raw data. All authors read and approved the final manuscript.

\section{Ethics approval and consent to participate}

Not applicable.

\section{Patient consent for publication}

Not applicable.

\section{Competing interests}

The authors declare that they have no competing interests.

\section{References}

1. Rudin CM, Brambilla E, Faivre-Finn C and Sage J: Small-cell lung cancer. Nat Rev Dis Primers 7: 3, 2021.

2. Huarte M: The emerging role of lncRNAs in cancer. Nat Med 21: $1253-1261,2015$

3. Gomes AQ, Nolasco S and Soares H: Non-coding RNAs: Multi-tasking molecules in the cell. Int J Mol Sci 14: 16010-16039, 2013.

4. Zhang J, Zhang P, Wang L, Piao PL and Ma L: Long non-coding RNA HOTAIR in carcinogenesis and metastasis. Acta Biochim Biophys Sin (Shanghai) 46: 1-5, 2014.

5. Cai B, Song XQ, Cai JP and Zhang S: HOTAIR: A cancer-related long non-coding RNA. Neoplasma 61: 379-391, 2014.

6. Qi X, Zhang DH, Wu N, Xiao JH, Wang X and Ma W: ceRNA in cancer: Possible functions and clinical implications. J Med Genet 52: 710-718, 2015.

7. Tam C, Wong JH, Tsui SKW, Zuo T, Chan TF and Ng TB: LncRNAs with miRNAs in regulation of gastric, liver, and colorectal cancers: Updates in recent years. Appl Microbiol Biotechnol 103: 4649-4677, 2019.

8. Liu XH, Sun M, Nie FQ, Ge YB, Zhang EB, Yin DD, Kong R, Xia R, Lu KH, Li JH, et al: Lnc RNA HOTAIR functions as a competing endogenous RNA to regulate HER2 expression by sponging miR-331-3p in gastric cancer. Mol Cancer 13: 92, 2014

9. Zhang S, Wang W, Liu G, Xie S, Li Q, Li Y and Lin Z: Long non-coding RNA HOTTIP promotes hypoxia-induced epithelial-mesenchymal transition of malignant glioma by regulating the miR-101/ZEB1 axis. Biomed Pharmacother 95: 711-720, 2017.

10. Yang B, Gao G, Wang Z, Sun D, Wei X, Ma Y and Ding Y: Long non-coding RNA HOTTIP promotes prostate cancer cells proliferation and migration by sponging miR-216a-5p. Biosci Rep 38 BSR20180566, 2018.

11. Sun Y, Hu B, Wang Q, Ye M, Qiu Q, Zhou Y, Zeng F, Zhang X, Guo $Y$ and Guo L: Long non-coding RNA HOTTIP promotes BCL-2 expression and induces chemoresistance in small cel lung cancer by sponging miR-216a. Cell Death Dis 9: 85, 2018.
12. Sun Y, Zhou Y, Bai Y, Wang Q, Bao J, Luo Y, Guo Y and Guo L: A long non-coding RNA HOTTIP expression is associated with disease progression and predicts outcome in small cell lung cancer patients. Mol Cancer 16: 162, 2017.

13. Niu Y, Ma F, Huang W, Fang S, Li M, Wei T and Guo L: Long non-coding RNA TUG1 is involved in cell growth and chemoresistance of small cell lung cancer by regulating LIMK $2 \mathrm{~b}$ via EZH2. Mol Cancer 16: 5, 2017.

14. Su F, Li H, Yan C, Jia B, Zhang Y and Chen X: Depleting MEKK1 expression inhibits the ability of invasion and migration of human pancreatic cancer cells. J Cancer Res Clin Oncol 135: 1655-1663, 2009.

15. Chen X, Han H, Li Y, Zhang Q, Mo K and Chen S: Upregulation of long noncoding RNA HOTTIP promotes metastasis of esophageal squamous cell carcinoma via induction of EMT. Oncotarget 7: 84480-84485, 2016.

16. Lin $\mathrm{C}$, Wang $\mathrm{Y}$, Wang $\mathrm{Y}$, Zhang $\mathrm{S}$, Yu L, Guo $\mathrm{C}$ and $\mathrm{Xu} \mathrm{H}$ : Transcriptional and posttranscriptional regulation of HOXA13 by lncRNA HOTTIP facilitates tumorigenesis and metastasis in esophageal squamous carcinoma cells. Oncogene 36: 5392-5406, 2017.

17. Han S, Jin X, Liu Z, Xing F, Han Y, Yu X, He G and Qiu F: The long noncoding RNA HOTTIP promotes breast cancer cell migration, invasiveness, and epithelial-mesenchymal transition via the Wnt- $\beta$-catenin signaling pathway. Biochem Cell Biol 97: 655-664, 2019.

18. Mu M, Li Y, Zhan Y, Li X and Zhang B: Knockdown of HOXA transcript at the distal tip suppresses the growth and invasion and induces apoptosis of oral tongue squamous carcinoma cells. Onco Targets Ther 11: 8033-8044, 2018.

19. Mao Z, Wu Y, Zhou J and Xing C: Salinomycin reduces epithelial-mesenchymal transition-mediated multidrug resistance by modifying long noncoding RNA HOTTIP expression in gastric cancer cells. Anticancer Drugs 30: 892-899, 2019.

20. Tang Y and Ji F: IncRNA HOTTIP facilitates osteosarcoma cell migration, invasion and epithelial-mesenchymal transition by forming a positive feedback loop with c-Myc. Oncol Lett 18: $1649-1659,2019$.

21. Fu Z, Chen C, Zhou Q, Wang Y, Zhao Y, Zhao X, Li W, Zheng S, Ye H, Wang L, et al: LncRNA HOTTIP modulates cancer stem cell properties in human pancreatic cancer by regulating HOXA9. Cancer Lett 410: 68-81, 2017.

22. Castro-Oropeza R, Melendez-Zajgla J, Maldonado V and Vazquez-Santillan K: The emerging role of lncRNAs in the regulation of cancer stem cells. Cell Oncol (Dordr) 41: 585-603, 2018.

23. Wang M,Zhang R, Zhang S, Xu R and Yang Q: MicroRNA-574-3p regulates epithelial mesenchymal transition and cisplatin resistance via targeting ZEB1 in human gastric carcinoma cells. Gene 700: 110-119, 2019.

24. Xiong L, Tang Y, Tang J, Liu Z and Wang X: Downregulation of lncRNA HOTTIP suppresses the proliferation, migration, and invasion of oral tongue squamous cell carcinoma by regulation of HMGA2-mediated Wnt/ $\beta$-catenin pathway. Cancer Biother Radiopharm 35: 720-730, 2020.

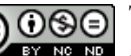

This work is licensed under a Creative Commons Attribution-NonCommercial-NoDerivatives 4.0 International (CC BY-NC-ND 4.0) License. 